\title{
A Rare Case of Chin Cutaneous Metastasis from Transitional Cell Carcinoma of Bladder in a Patient who Underwent Radical Cystectomy
}

\author{
Giovanni Luca Giliberto*, Carmelo Agostino Di Franco and Bruno Rovereto \\ Department of Urology IRCC "San Matteo" Pavia, Italy
}

Submission: November 11, 2016; Published: December 12, 2016

*Corresponding author: Giovanni Luca Giliberto, Department of Urology IRCC “San Matteo” Pavia, Italy, Email: gl.giliberto@gmail.com

\begin{abstract}
Facial cutaneous metastasis from primary bladder cancers is very rare and they are usually associated with poor prognosis. In literature, very few cases of cutaneous metastasis from urothelial malignancies are reported but we did not find any case of bladder cancer metastases localised to chin; usually cutaneous metastasis from bladder are localised in chest or scrotal skin. We report a 66 years-old patient affected by transitional muscle-invasive cell cancer of bladder who underwent radical cystectomy and who developed in two months after operation a skin lesion localised to chin. Clinical stage of bladder cancer was cT2 N0 M0; the patient was studied before operation through total body CT-scan, TB bone-scan negative for metastasis. The patient was totally asymptomatic and he had a good performance status. We discuss the need to have suspicion in presence of cutaneous lesions, apparently benign, in a patient affected by malignancy in order to start quickly a proper treatment.
\end{abstract}

\section{Case Report}

A 66-year old Caucasian man underwent radical cystectomy for transitional muscle-invasive cell cancer of bladder with orthotopic neobladder reconstruction. We made the diagnosis of cancer through a TURB performed around one month before. It showed a transitional cell cancer (TCC) of bladder, high grade (WHO 2004) G2-G3 (WHO 1973). The patient was asymptomatic and he had a good performance status. Histological examination after radical cystectomy reported a TCC muscle-invasive, high grade (WHO 2004), G3 (WHO 1973), mainly localised in right bladder wall (Figure 1). The cancer infiltrates all the bladder wall thickness. Microscopically, it reaches the perivesical fat tissue (pT3a) with images of perineural neoplastic permeation. The remaining mucosa was affected by CIS (carcinoma in situ). All the lymph nodes were negative for metastasis ( $\mathrm{pN} 0)$.

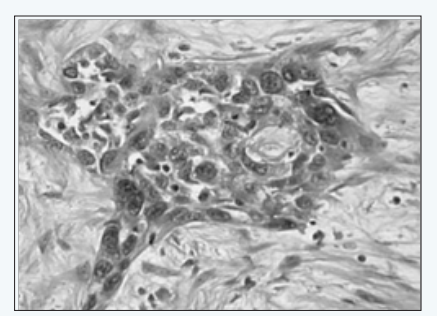

Figure 1: Infiltration of bladder wall by a high grade G3 transitional cells carcinoma (TCC).
After around one month, during follow-up controls, the patient was evaluated in our department for one single tender purple nodule localised at the chin. Initially, the lesion was thought to be a furuncle. It continued to enlarge with objective signs of phlogosis and relative lack of pain. In this moment, the patient was totally asymptomatic. We required a maxillofacial surgery consultation. The patient performed a punch biopsy of skin lesion that showed a metastatic carcinoma, histologically identical to the primary bladder cancer. Microscopically, it showed pleomorphic hyperchromatic cells with high mitotic activity and large nucleoli in dermis and epidermis (Figure 2). We performed a total body CT-scan that revealed bone metastasis of lumbosacral column. We sent the patient to the maxillofacial surgeon to remove the pathological nodule and to the oncological evaluation in order to do adjuvant chemotherapy.

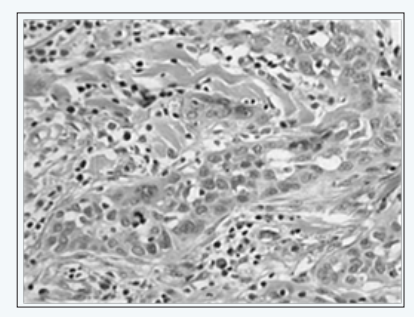

Figure 2: Skin biopsy showing metastatic TCC analogous to the primary bladder cancer. 


\section{Discussion}

In literature, the overall incidence of cutaneous metastasis from primary solid visceral cancers is low, around 2,9 and $5,3 \%$; the most common site of metastasis is chest and the most common primary cancer is breast [1]. Cutaneous metastasis from transitional cells cancers are rare and bladder represents the primary cancer in $0,84 \%$ to $3,6 \%$ of cases [2]. In literature, cases of cutaneous metastases from TCC bladder cancer are described $[3,4]$ but we did not find any case of facial metastasis, in particular to chin, from bladder cancer. Usually, presence of cutaneous metastasis is associated with poor prognosis, but in literature some cases of regression are described [5]. Cutaneous metastasis could be taken for other benign cutaneous disorders such as sebaceous cysts or furuncles, but in presence of cutaneous lesion with phlogosis signs, lack or low pain and tendence to enlarge and ulcerate it is necessary to put the suspect of a metastatic lesion. Cutaneous metastases from bladder TCC usually develop in locoregional skin in particular abdomen, genitalia and tights and it is supposed to be related to lymphatic spread [6]. In our case, chin localisation could be related to hematogenous spread. We think that histological examination of radical cystectomy could predict a high index risk that a cutaneous lesion could be metastasis; in particular, the stage $\mathrm{T} 2$ or superior, the presence of a carcinoma in situ and the perineural neoplastic permeation. In our case, lymph nodes were negatives (NO disease) but we had a rapid progression of the disease with cutaneous metastases and bone metastases (lumbosacral column) in a patient totally asymptomatic. We think that cutaneous metastases are frequently associated with other more common sites of metastasis, such as bone and visceral metastasis, and for this we need to keep in consideration to start precociously a total body staging of the patient.

\section{References}

1. Krathen RA, Orengo IF, Rosen T (2003) Cutaneous metastasis: a metaanalysis of data. South Med. J 96(2): 164-167.

2. Mueller TJ, Wu H, Greenberg RE, Hudes G, Topham N, et al. (2004) Cutaneous metastases from genitourinary malignancies. Urology 63(6): 1021-1026.

3. Eardley I, Abercrombie J, Wright BJ, Hendry WF (1987) Cystic cutaneous metastases from transitional cell carcinoma of the bladder. J R Soc Med 80(5): 314-315.

4. Safer LF, Pirozzi DJ (1980) Extensive cutaneous metastases from urinary bladder carcinoma. Cutis 26(5): 500.

5. Mancebo JM, de la Peña J, Hidalgo L, Cisneros J, Machuca J, et al. (1985) [Spontaneous regression of cutaneous metastases of transitional cell carcinoma of the bladder]. Arch Esp Urol 38(5): 497501.

6. Zwenger EM, Kaatz M, Ziemer M (2006) Skin metastates of "nested type" urothelial carcinoma of the urinary bladder. J Cutan Pathol 33(1):

754-755. 